\title{
Trends and Advances in Operando Methodology
}

\author{
Atsushi Urakawa*
}

\begin{abstract}
After the introduction of the term, operando, the catalysis community has taken significant steps forward to understand chemistry and physics taking place within catalyst body and catalytic reactor on different length and time scales with great motivation to firmly establish catalyst structure vs. catalytic performance relationships. Herein recent advances, current trends and possible future directions in operando methodology are briefly summarized.
\end{abstract}

\section{Introduction}

Nearly 15 years has passed since the Latin word, operando, was coined by Miguel A. Bañares, Eric M. Gaigneaux, Gerhard Mestl, and Bert M. Weckhuysen to distinguish the experimental conditions from in situ and the first papers mentioning operando appeared in 2002 [1-4]. As described in detail by Bañares [5] and Weckhuysen [6], this historical action was motivated by the necessity to highlight the importance of studying catalyst materials under working conditions (i.e. showing practically relevant catalytic activity) by analytical and visualization techniques. The definition of catalysts' working condition naturally requires verification of catalytic activity and selectivity. Hence, the condition of in situ study of catalyst materials and/or catalytically active species together with simultaneous evaluation of catalytic performance is generally defined as operando methodology. The term has now widespread to various research fields such as gas sensors [7,8] and battery [9-11] when 
spectroscopic measurements and spectro-imaging are performed under technologically relevant conditions to elucidate structure-performance relationships.

The birth of operando methodology in catalysis was urged by the increasing number of examples showing large temperature, pressure and materials gaps where catalytically active species and sites could be uniquely formed under specific reaction conditions, typically a combination of high-pressure, high-temperature, and specific nano-structured catalyst materials deposited over inorganic materials which are shaped in a pellet form (Figure 1). Nowadays, it is widely recognized that the structure of catalyst surface is dynamic and often undergoes reconstruction when reactants interact with the outer surface. A notable example is the atmospheric-pressure STM study of CO oxidation by Hendriksen and Frenken [12]. When the partial pressure of $\mathrm{O}_{2}$ to $\mathrm{CO}$ was sufficiently high, the Pt surface was reconstructed, forming $\mathrm{PtO}_{2}$-like islands which were remarkably more active in $\mathrm{CO}$ oxidation than the clean Pt surface $[13,14]$. When $\mathrm{O}_{2}$ concentration became low, the Pt surface reconstructed back to flat Pt surface, showing the dynamic nature of the catalytic surface.[12,15] Similar insights were also gained for Pd surface and the widely observed oscillatory behavior of CO oxidation activity could be elegantly explained by formation and disappearance of the highly active nano-oxide phase of Pd.[16] These studies show that well-defined materials under operando conditions can yield precise and important physical and chemical insights on the atomic scale.

Obtaining the key information on catalyst materials and reaction conditions directly linked to the reactivity of catalysts on the laboratory or industrial scale requires, however, further efforts in simulating and understanding the complexity of the catalyst material, diffusional characteristics, and hydrodynamics on different length scales, i.e. those of atom, catalyst particle, pellet and reactor $[17,18]$. When catalyst pellets are packed in a reactor and a fluid of reactant(s) passes through it, there are always concentration gradients of reactants, 
products and possible intermediates in the fluid and over catalyst surface along the axial and possibly radial direction of the reactor. On a smaller scale, within the catalyst pellet, reactions take place and concentration gradients of reactants, intermediates and products arise. On the same scale, elemental and concentrational heterogeneities of catalytically active materials within the pellet may be present deliberately or accidentally. On the smallest scale, catalytic activity is induced and influenced by various physical and chemical phenomena such as atomic/molecular adsorption, surface reaction, desorption, surface reconstruction, active site formation, and/or surface poisoning, which also create concentrational heterogeneities because actual catalyst surfaces are never flat like perfect single crystals but complexly shaped mountains and valleys composed of various chemical elements. It is important to realize that the above gradients on the scales of reactor and catalyst pellet can directly influence the atomic scale surface chemistry because they impact on the concentrations of chemical species in the fluid phase, which can be even time-dependent [19]. Moreover, temperature may vary on each length scales and understanding these gradients can be of direct relevance for full comprehension of heterogeneous catalytic processes [20].

Furthermore, even when technology allows studying physicochemical processes on the different length and desired time scales, there is another issue of studying catalytically active sites and species selectively under operando conditions without the information buried in the overwhelming signals of spectator chemical species, solvent and catalyst support materials. This is inherent to any measurement techniques and becomes serious under operando conditions, where surface chemical species are present at high concentrations and selection of detection methods is limited by the typical operational conditions of high temperature and pressure.

Over the past years, significant progresses were made to tackle the above challenging aspects of operando methodology and catalysis research in general. Recent trends and 
advances made in the field from my personal view are briefly highlighted in Figure 2 and summarized below.

\section{Multi-probe approach}

Using a single characterization technique often fails to give a sufficiently precise picture of catalytic processes and materials because it is limited in probing area (spaceresolution), speed (time-resolution), transition (type of excitation/relaxation), element, electronic structure and/or geometrical structure (long/short-range ordering). Therefore, combined use of complementary techniques is desired to gain holistic views on catalytic processes (Figure 2a) [21]. Powerful combination to gain molecular, electronic and structural information is vibrational (IR/Raman) spectroscopy combined with X-ray spectroscopy/diffraction [22]. Newton has pioneered the combination of diffuse reflectance Fourier transform infrared spectroscopy (DRIFTS) and X-ray absorption spectroscopy (XAS), the former providing information about chemical bonds of surface species and the latter providing geometrical and electronic structures of active metals [23]. The advent of low-noise, high-resolution 2D detectors has enabled X-ray diffraction (XRD) to be combined with other techniques like XAS using the same capillary reactor cell [21,22,24,25]. Another combination gaining popularity recently using X-ray is XAS/X-ray emission spectroscopy (XES), which can yield a detailed picture of electronic states (unoccupied and occupied orbitals). Also, local coordination (e.g. adsorbate or ligand) environment can be studied by the latter technique more precisely. An elegant use of the combination was recently demonstrated by Jacob and Grunwaldt to elucidate the chemistry around active Fe center located in a zeolite structure for selective catalytic reduction (SCR) of $\mathrm{NO}$ by $\mathrm{NH}_{3}$ [26]. Possibilities for the combination of operando probes are infinite and it is important to choose the most appropriate techniques to investigate the key information under question. 


\section{Space-resolved spectroscopy/diffraction, imaging and tomography}

Seeing the reality by eyes (with the help of light) is often worth more than a number of catalytic experiments. This approach is very rapidly evolving and has been recently demonstrated by several research groups under operando conditions. Generally, three different modes are used to add space-resolution to a technique on reactor, pellet and atomic scale with/without the help of post-mathematical reconstruction of images.

The first one (Figure 2b) makes use of a focused light and one gains a spaceresolution by moving a pellet/reactor. Confocal Raman spectroscopy operates in this mode and has been applied to study water transport within proton-conducting polymer electrolyte under operando condition [27]. Along entire catalyst beds, operando space- and timeresolved DRIFTS-Raman [19], XAFS [28] and XRD [29] were used to study catalyst materials in deNO $\mathrm{x}_{\mathrm{x}}$ chemistry and methanol-to-olefin (MTO) process. The second mode (Figure 2c) is to obtain the full picture in one shot using a 2D detector with a great advantage in the time-resolution [30]. Such a fast 2D detector was used also for single-molecule counting based on total internal reflection fluorescence microscopy (TIRFM) to prove that a cycloaddition reaction took place over the surface of $\mathrm{Cu}$ nanoparticles and not in the solution by possible leached $\mathrm{Cu}$ species [31].

The third mode (Figure 2d) for chemical/structural imaging or tomography, slice by slice, is widely used in magnetic resonance imaging (MRI) well known for medical applications. MRI is also actively employed in heterogeneous catalysis research by Koptyug to gain unique insights into mass transport and heat transfer on the pellet and reactor scales [32]. Similar slicing approach using computed tomography (CT) of XRD, XAS, and X-ray fluorescence (XRF) have been pioneered by Beale, Jacques, Di Michiel, and Weckhuysen among others using mainly synchrotron facilities [33,34] and even in the lab [35]. Inside the complex membrane reactors for oxidative coupling of methane (OCM) reaction could be seen 
through [36] and in another study even a catalyst particle in liquid phase chemistry could be studied [37]. Impressive time-resolution $(>1 \mathrm{~Hz})$ was demonstrated in battery research under operando thermal runaway using CT-tomography and radiography combined with thermal imaging [38].

\section{Multivariate analysis}

Analysis of operando spectroscopic data often suffers from three issues. One is related to the detection selectivity towards what we would like to hunt as described above. Another challenge is the interpretation of spectra, which is often hindered by overlapping signals of coexisting multiple components in the bulk and/or on the surface of catalyst solids. Under operando conditions, the situation is significantly more challenging compared to that under well-defined UHV conditions. Even worse, appropriate reference spectra are often unavailable because catalytically active species and phases may be only present stably or transiently under operando conditions.

The problem of detection selectivity may be elegantly solved by exerting a perturbation of an external parameter (temperature, concentration, light flux, etc.) to a catalytic system such that the reaction rates and concentrations of species/phase of interest vary. Such step/pulse-response experiments are widely used. Among them, steady-state isotopic transient kinetic analysis (SSITKA) is an experimental technique to follow the kinetics of catalytic reactions by varying reactant concentration in the form of isotopomers without disturbing the chemistry [40]. Another approach to add selectivity with drastic improvement in detection sensitivity is modulation excitation spectroscopy (MES) [41]. MES uses periodic change of an external parameter (e.g. varying reactant concentration, ideally from that of steady-state one for operando studies). Spectra are continuously measured over a number of stimulus-relaxation cycles and averaged into one cycle (first $\mathrm{S} / \mathrm{N}$ improvement) 
and then the one-cycle data are further processed by phase-sensitive detection (PSD) to filter out static signal (e.g. due to solvent and catalyst support) and noise (second $\mathrm{S} / \mathrm{N}$ improvement) and to kinetically differentiate evolving signals in the so-called phase domain [41]. MES can be combined with any techniques such as IR [42] and XAFS [43]. Recently, the theory of MES for diffraction has been developed and the technique is called modulation enhanced diffraction (MED) [44]. By MED one can study active sublattices [45] and element-specificity can be achieved by applying X-ray energy MED [46].

SSITKA and MES can extract valuable information, but both often suffer from signal overlaps and occasional unavailability of spectral references for interpretation. Recently, an increasing number of applications of multivariate spectral analysis suited for blind source separation has been witnessed. In such analysis, one measures spectra over a period of time with/without external perturbation and directly post-process the spectroscopic data by the mathematical engine to separate them into kinetically pure components and respective concentration profiles (Figure 2e). Blind means there is no reference required for the spectral separation process. Band-target entropy minimization (BTEM) actively applied in homogeneous catalysis [47] was also employed to identify surface species measured by DRIFTS during the reaction of $\mathrm{CO}$ and NO over Pd [48]. Non-negative matrix factorization (NNMF) was used to separate overlapping UV-Vis spectra to identify deactivating species during MTO process over H-SSZ-13 [49]. Multivariate curve resolution (MCR) became very popular across different disciplines for blind source separation and it has been successfully used for XAS [50,51] and IR spectroscopy [52] with kinetic analysis of heterogeneous catalytic reactions with a strong emphasis to simplify the spectral analysis by the conversion of numerous spectra to a few to some component spectra and respective concentration profiles.

\section{Outlook}


Categorizing specific areas of in situ into operando has promoted the awareness of researchers about the importance of operando methodology in catalysis research to study catalysts under technologically relevant conditions and to look at important spots within catalytic reactors and catalyst materials. The intentional use of the term, operando, urged by the awareness of the "gaps" has obviously driven the great technological advances in demanding and sophisticated space- and time-resolved measurements on different length scales. There are clear next steps in the three main streams of operando methodology described here by employing more complementary probes measured simultaneously, enhancing further the space/time resolution, and improving the blind source spectral separation methods. Yet, the challenges of operando community still remain as indicated by the generally poor penetration of operando methodology in industry or by scarce collaboration between academic and industry researchers using operando methodology from the author's view. The point is less scientific but more opportunities for dialogs between researchers in academia and industry are crucial to enhance the relevance of research work to know exactly what kind of aspects are more relevant in industrial processes and to know conditional constraints in commercial processes. Along the same line, the relevance is best ascertained when operando studies are performed in a reactor where catalytic performance makes practical sense. For this, chemical engineering aspects like hydrodynamics, mass transport, and reaction kinetics are ideally taken into account in operando spectroscopic reactor design. These aspects are actually best clarified by also operando methodologies using spectroscopic and visual inspection to see through operando reactor mimicking industrial processes or envisioned future catalytic processes.

\section{Acknowledgements}

MINECO is acknowledged for support through Severo Ochoa Excellence Accreditation 2014-2018 (SEV-2013-0319) and CTQ2012-34153. 


\section{References}

1. Bañares MA, Guerrero-Pérez MO, Fierro JLG, Cortez GG: Raman spectroscopy during catalytic operations with on-line activity measurement (operando spectroscopy): a method for understanding the active centres of cations supported on porous materials. $J$ Mater Chem (2002) 12(11):3337-3342.

2. Bañares MA, Wachs IE: Molecular structures of supported metal oxide catalysts under different environments. J Raman Spectrosc (2002) 33(5):359-380.

3. Guerrero-Pérez MO, Bañares MA: Operando Raman study of alumina-supported Sb-V-O catalyst during propane ammoxidation to acrylonitrile with on-line activity measurement. Chem Commun (2002) 12):1292-1293.

4. Weckhuysen BM: Snapshots of a working catalyst: possibilities and limitations of in situ spectroscopy in the field of heterogeneous catalysis. Chem Commun (2002) 2):97-110.

** Comprehensive summary of essense and importance of operando methodology from various examples, although the term operando was only mentioned as one of possible candidates at that time.

5. Bañares MA: Operando methodology: combination of in situ spectroscopy and simultaneous activity measurements under catalytic reaction conditions. Catal Today (2005) 100(1-2):71-77.

** Clearly defining operando methodology currently used in catalysis communitry.

6. Weckhuysen BM: Determining the active site in a catalytic process: Operando spectroscopy is more than a buzzword. Phys Chem Chem Phys (2003) 5(20):4351-4360.

** Discussing the potential and limitaions of operando methodology with a stimulating perspective of future developemnt of the field more than 10 years ago, which clearly guided its later development.

7. Koziej D, Thomas K, Barsan N, Thibault-Starzyk F, Weimar U: Influence of annealing temperature on the $\mathrm{CO}$ sensing mechanism for tin dioxide based sensors - Operando studies. Catal Today (2007) 126(1-2):211-218.

8. Sänze S, Gurlo A, Hess C: Monitoring Gas Sensors at Work: Operando RamanFTIR Study of Ethanol Detection by Indium Oxide. Angew Chem Int Ed (2013) 52(13):3607-3610.

9. Nelson J, Misra S, Yang Y, Jackson A, Liu Y, Wang H, Dai H, Andrews JC, Cui Y, Toney MF: In Operando X-ray Diffraction and Transmission X-ray Microscopy of Lithium Sulfur Batteries. J Am Chem Soc (2012) 134(14):6337-6343.

10. Gu M, Parent LR, Mehdi BL, Unocic RR, McDowell MT, Sacci RL, Xu W, Connell JG, Xu P, Abellan $\mathrm{P}$, Chen $\mathrm{X}$ et al: Demonstration of an Electrochemical Liquid Cell for Operando Transmission Electron Microscopy Observation of the Lithiation/Delithiation Behavior of Si Nanowire Battery Anodes. Nano Lett (2013) 13(12):6106-6112. 
11. Li L, Chen-Wiegart Y-cK, Wang J, Gao P, Ding Q, Yu Y-S, Wang F, Cabana J, Wang J, Jin S: Visualization of electrochemically driven solid-state phase transformations using operando hard X-ray spectro-imaging. Nat Commun (2015) 6:6883.

12. Hendriksen BLM, Frenken JWM: CO oxidation on Pt(110): Scanning tunneling microscopy inside a high-pressure flow reactor. Phys Rev Lett (2002) 89(4):046101.

* Elegant work to visualize a reversible surface reconstraction of Pt under operando condition by high-pressure STM.

13. Ackermann MD, Pedersen TM, Hendriksen BLM, Robach O, Bobaru SC, Popa I, Quiros C, Kim $\mathrm{H}$, Hammer B, Ferrer S, Frenken JWM: Structure and reactivity of surface oxides on Pt(110) during catalytic CO oxidation. Phys Rev Lett (2005) 95(25):255505.

14. Butcher DR, Grass ME, Zeng Z, Aksoy F, Bluhm H, Li W-X, Mun BS, Somorjai GA, Liu Z: In Situ Oxidation Study of $\mathrm{Pt}(\mathbf{1 1 0})$ and Its Interaction with CO. J Am Chem Soc (2011) 133(50):20319-20325.

15. van Spronsen MA, van Baarle GJC, Herbschleb CT, Frenken JWM, Groot IMN: High-pressure operando STM studies giving insight in $\mathrm{CO}$ oxidation and NO reduction over $\mathrm{Pt}(110)$. Catal Today (2015) 244:85-95.

16. Hendriksen BLM, Bobaru SC, Frenken JWM: Oscillatory CO oxidation on $\mathrm{Pd}(\mathbf{1 0 0})$ studied with in situ scanning tunneling microscopy. Surf Sci (2004) 552(1-3):229-242.

17. Weckhuysen BM: Chemical Imaging of Spatial Heterogeneities in Catalytic Solids at Different Length and Time Scales. Angew Chem Int Ed (2009) 48(27):4910-4943.

* Review on space-resolved spectroscopic techniques through representitive examples on different length and time scales.

18. Urakawa A, Baiker A: Space-Resolved Profiling Relevant in Heterogeneous Catalysis. Top Catal (2009) 52(10):1312-1322.

* Review on space-resolved spectroscopic techniques from methodological perpectives.

19. Urakawa A, Maeda N, Baiker A: Space- and Time-Resolved Combined DRIFT and Raman Spectroscopy: Monitoring Dynamic Surface and Bulk Processes during $\mathrm{NO}_{x}$ Storage Reduction. Angew Chem Int Ed (2008) 47(48):9256-9259.

20. Jarenwattananon NN, Glöggler S, Otto T, Melkonian A, Morris W, Burt SR, Yaghi OM, Bouchard L-S: Thermal maps of gases in heterogeneous reactions. Nature (2013) 502(7472):537-540.

21. Bentrup U: Combining in situ characterization methods in one set-up: looking with more eyes into the intricate chemistry of the synthesis and working of heterogeneous catalysts. Chem Soc Rev (2010) 39(12):4718-4730.

* Summarizing various common spectroscopic tools used in heterogeneous catalysis for the multiprobe approach. 
22. Newton MA, van Beek W: Combining synchrotron-based X-ray techniques with vibrational spectroscopies for the in situ study of heterogeneous catalysts: a view from a bridge. Chem Soc Rev (2010) 39(12):4845-4863.

* Describing the characteristics and advantages of the powerful combination of X-ray spectroscopy with vibrational spectroscopy.

23. Newton MA, Belver-Coldeira C, Martínez-Arias A, Fernández-García M: Dynamic in situ observation of rapid size and shape change of supported Pd nanoparticles during CO/NO cycling. Nature Materials (2007) 6(7):528-532.

24. Frenkel Al, Wang Q, Marinkovic N, Chen JG, Barrio L, Si R, López Cámara A, Estrella AM, Rodriguez JA, Hanson JC: Combining X-ray Absorption and X-ray Diffraction Techniques for in Situ Studies of Chemical Transformations in Heterogeneous Catalysis: Advantages and Limitations. J Phys Chem C (2011) 115(36):17884-17890.

25. Staniuk M, Hirsch O, Kränzlin N, Böhlen R, van Beek W, Abdala PM, Koziej D: Puzzling Mechanism behind a Simple Synthesis of Cobalt and Cobalt Oxide Nanoparticles: In Situ Synchrotron X-ray Absorption and Diffraction Studies. Chem Mater (2014) 26(6):2086-2094.

26. Boubnov A, Carvalho HWP, Doronkin DE, Guenter T, Gallo E, Atkins AJ, Jacob CR, Grunwaldt J-D: Selective Catalytic Reduction of NO Over Fe-ZSM-5: Mechanistic Insights by Operando HERFD-XANES and Valence-to-Core X-ray Emission Spectroscopy. J Am Chem Soc (2014) 136(37):13006-13015.

* Study on SCR, clearly exemplifying the powerful combination of XAS and XES for operando studies.

27. Deabate S, Huguet P, Morin A, Gebel G, Lanteri Y, Peng Z, Sutor AK: Raman Microspectroscopy as a Useful Tool for In Situ and Operando Studies of Water Transport in Perfluorosulfonic Membranes for PEMFCs. Fuel Cells (2014) 14(5):677-693.

28. Doronkin DE, Casapu M, Günter T, Müller O, Frahm R, Grunwaldt J-D: Operando Spatiallyand Time-Resolved XAS Study on Zeolite Catalysts for Selective Catalytic Reduction of $\mathbf{N O}_{\mathbf{x}}$ by $\mathrm{NH}_{3}$. J Phys Chem C (2014) 118(19):10204-10212.

29. Wragg DS, Brien MGO, Bleken FL, Di Michiel M, Olsbye U, Fjellvag $\mathrm{H}$ : Watching the Methanol-to-Olefin Process with Time- and Space-Resolved High-Energy Operando X-ray Diffraction. Angew Chem Int Ed (2012) 51(32):7956-7959.

30. Grunwaldt J-D, Schroer CG: Hard and soft X-ray microscopy and tomography in catalysis: bridging the different time and length scales. Chem Soc Rev (2010) 39(12):4741-4753.

31. Decan MR, Impellizzeri S, Luisa Marin M, Scaiano JC: Copper nanoparticle heterogeneous catalytic 'click' cycloaddition confirmed by single-molecule spectroscopy. Nat Commun (2014) 5:4612

32. Lysova AA, Koptyug IV: Magnetic resonance imaging methods for in situ studies in heterogeneous catalysis. Chem Soc Rev (2010) 39(12):4585-4601.

* Introduction to and review on the use of MRI in heterogeneous catalysis research and the unique information gained. 
33. Beale AM, Jacques SDM, Gibson EK, Di Michiel M: Progress towards five dimensional diffraction imaging of functional materials under process conditions. Coordin Chem Rev (2014) 277:208-223.

* Summarizing the practical aspects of multi-dimensional X-ray diffraction imaging.

34. Gonzalez-Jimenez ID, Cats K, Davidian T, Ruitenbeek M, Meirer F, Liu Y, Nelson J, Andrews JC, Pianetta P, de Groot FMF, Weckhuysen BM: Hard X-ray Nanotomography of Catalytic Solids at Work. Angew Chem Int Ed (2012) 51(48):11986-11990.

* The first use of full-field transmission hard X-ray microscopy for element- and structure-specific nanotomography of catalyst materials under working condition.

35. Egan CK, Jacques SDM, Wilson MD, Veale MC, Seller P, Beale AM, Pattrick RAD, Withers PJ, Cernik RJ: 3D chemical imaging in the laboratory by hyperspectral X-ray computed tomography. Scientific Reports (2015) 5:15979.

36. Vamvakeros A, Jacques SDM, Middelkoop V, Di Michiel M, Egan CK, Ismagilov IZ, Vaughan GBM, Gallucci F, Annaland MvS, Shearing PR, Cernik RJ et al: Real time chemical imaging of a working catalytic membrane reactor during oxidative coupling of methane. Chem Commun (2015) 51(64):12752-12755.

37. Price SWT, Geraki K, Ignatyev K, Witte PT, Beale AM, Mosselmans JFW: In Situ Microfocus Chemical Computed Tomography of the Composition of a Single Catalyst Particle During Hydrogenation of Nitrobenzene in the Liquid Phase. Angew Chem Int Ed (2015) 54(34):9886-9889.

38. Finegan DP, Scheel M, Robinson JB, Tjaden B, Hunt I, Mason TJ, Millichamp J, Di Michiel M, Offer GJ, Hinds G, Brett DJL et al: In-operando high-speed tomography of lithium-ion batteries during thermal runaway. Nat Commun (2015) 6:6924

39. Li Y, Zakharov D, Zhao S, Tappero R, Jung U, Elsen A, Baumann P, Nuzzo RG, Stach EA, Frenkel Al: Complex structural dynamics of nanocatalysts revealed in Operando conditions by correlated imaging and spectroscopy probes. Nat Commun (2015) 6:6883.

40. Ledesma C, Yang J, Chen D, Holmen A: Recent Approaches in Mechanistic and Kinetic Studies of Catalytic Reactions Using SSITKA Technique. ACS Catal (2014) 4(12):4527-4547.

* Introduction to the methodology of SSITKA and reviews on mechanistic studies using SSITKA.

41. Urakawa A, Bürgi T, Baiker A: Sensitivity enhancement and dynamic behavior analysis by modulation excitation spectroscopy: Principle and application in heterogeneous catalysis. Chem Eng Sci (2008) 63(20):4902-4909.

* Tutorial introduction to modulation excitation spectroscopy (MES).

42. Bürgi T, Baiker A: In situ infrared spectroscopy of catalytic solid-liquid interfaces using phase-sensitive detection: Enantioselective hydrogenation of a pyrone over $\mathrm{Pd} / \mathrm{TiO}_{2}$. J Phys Chem B (2002) 106(41):10649-10658. 
43. Marchionni V, Newton MA, Kambolis A, Matam SK, Weidenkaff A, Ferri D: A modulated excitation ED-EXAFS/DRIFTS study of hydrothermal ageing of $\mathrm{Rh} / \mathrm{Al}_{2} \mathrm{O}_{3}$. Catal Today (2014) 229:80-87.

44. Chernyshov D, van Beek W, Emerich H, Milanesio M, Urakawa A, Viterbo D, Palin L, Caliandro $\mathrm{R}$ : Kinematic diffraction on a structure with periodically varying scattering function. Acta Crystallogr A (2011) 67:327-335.

45. Ferri D, Newton MA, Di Michiel M, Chiarello GL, Yoon S, Lu Y, Andrieux J: Revealing the Dynamic Structure of Complex Solid Catalysts Using Modulated Excitation X-ray Diffraction. Angew Chem Int Ed (2014) 53(34):8890-8894.

46. van Beek W, Emerich H, Urakawa A, Palin L, Milanesio M, Caliandro R, Viterbo D, Chernyshov $D$ : Untangling diffraction intensity: modulation enhanced diffraction on $\mathrm{ZrO}_{2}$ powder. $J$ Appl Cryst (2012) 45:738-747.

47. Garland M: Combining operando spectroscopy with experimental design, signal processing and advanced chemometrics State-of-the-art and a glimpse of the future. Catal Today (2010) 155(3-4):266-270.

48. Chilukoti S, Gao F, Anderson BG, Niemantsverdriet JWH, Garland M: Pure component spectral analysis of surface adsorbed species measured under real conditions. BTEMDRIFTS study of $\mathrm{CO}$ and $\mathrm{NO}$ reaction over a $\mathrm{Pd} / \mathrm{p}-\mathrm{Al}_{2} \mathrm{O}_{3}$ catalyst. Phys Chem Chem Phys (2008) 10(36):5510-5520.

49. Borodina E, Meirer F, Lezcano-González I, Mokhtar M, Asiri AM, Al-Thabaiti SA, Basahel SN, Ruiz-Martinez J, Weckhuysen BM: Influence of the Reaction Temperature on the Nature of the Active and Deactivating Species during Methanol to Olefins Conversion over H-SSZ-13. ACS Catal (2015) 5(2):992-1003.

50. Voronov A, Urakawa A, van Beek W, Tsakoumis NE, Emerich H, Rønning M: Multivariate curve resolution applied to in situ X-ray absorption spectroscopy data: An efficient tool for data processing and analysis. Anal Chim Acta (2014) 840:20-27.

51. Cassinelli WH, Martins L, Passos AR, Pulcinelli SH, Santilli CV, Rochet A, Briois V: Multivariate curve resolution analysis applied to time-resolved synchrotron X-ray Absorption Spectroscopy monitoring of the activation of copper alumina catalyst. Catal Today (2014) 229:114-122.

52. Fakhrnasova D, Chimentão RJ, Medina F, Urakawa A: Rational and Statistical Approaches in Enhancing the Yield of Ethylene Carbonate in Urea Transesterification with Ethylene Glycol over Metal Oxides. ACS Catal (2015) 5(11):6284-6295.

* An example where multivariate analysis was used for spectral deconvolution and to study the kinetics of heterogeneously catalyzed reactions. 
Figure 1. Three major gaps (material gap, temperature gap and pressure gap) often causing problems in practical relevance of spectroscopic and diffraction studies of catalyst materials. These gaps have to be filled to address real situation of catalyst material at work under technologically relevant conditions. UHV stands for ultra-high vacuum.

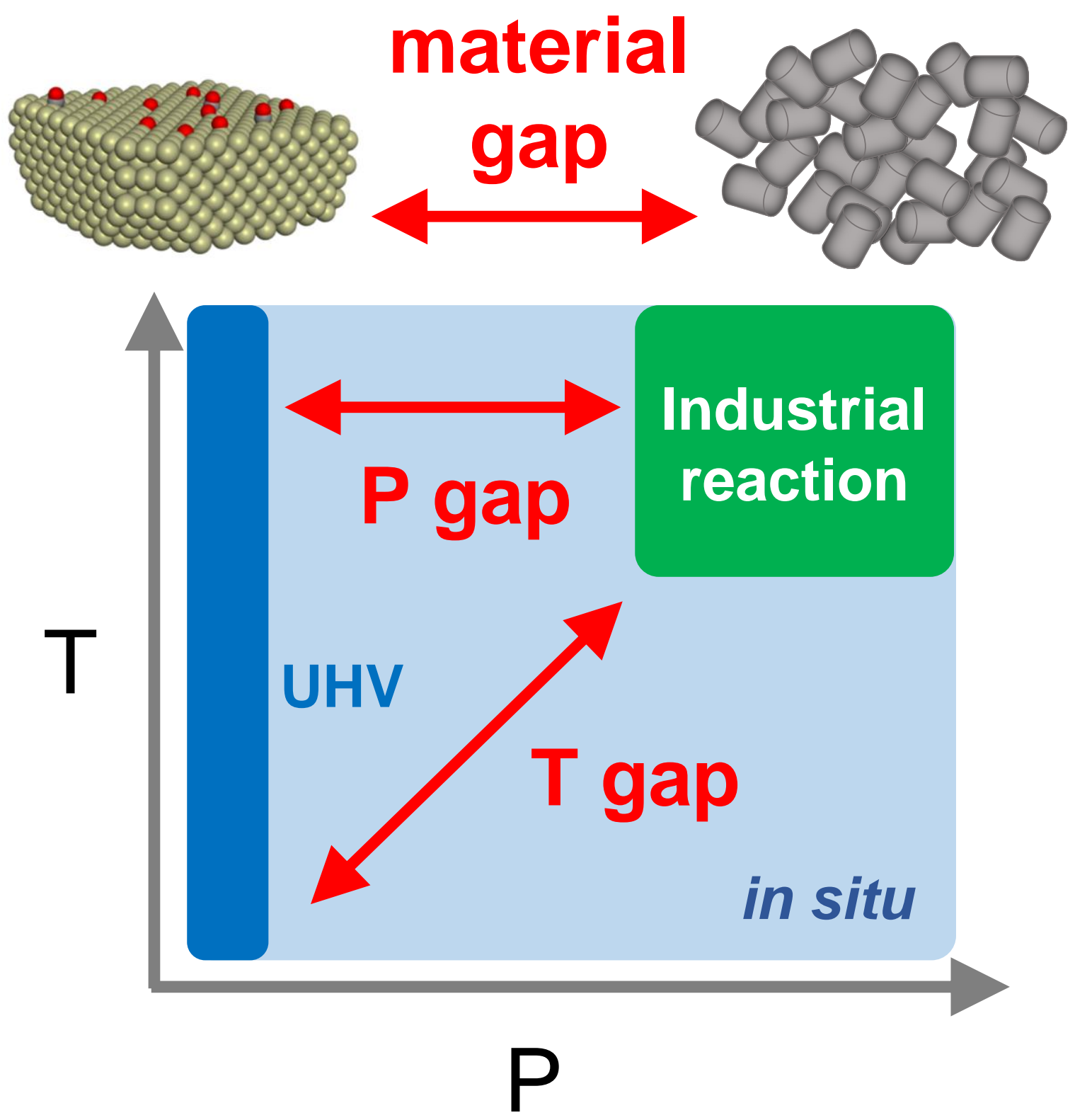


Figure 2. Trends in operando methodology: (a) multi-probe approach, (b-d) space- and possibly time-resolved approach, where spatial resolution is achieved by (b) moving a sample (reactor/catalyst particle), (c) using 2D detector, (d) first sliced 2D then 3D spectroimaging by sample translation, and (e) multivariate analysis to identify spectroscopically "pure" components and their concentration profiles often without the need of reference sample measurements. For (a-d) the approach can be used for studying a single catalyst particle or pellet.

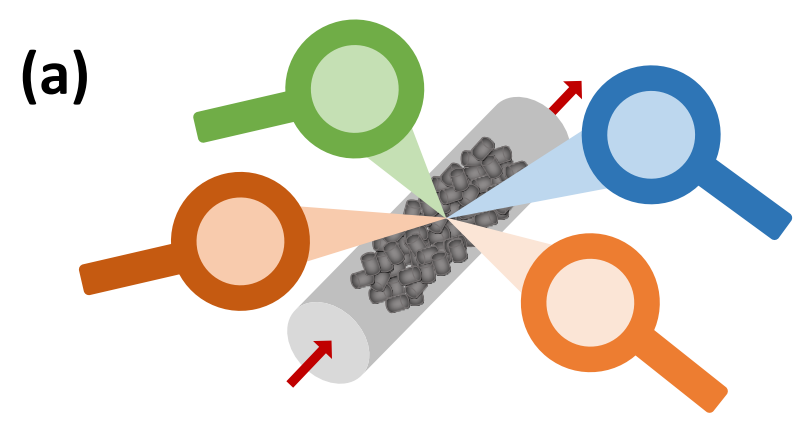

(b)

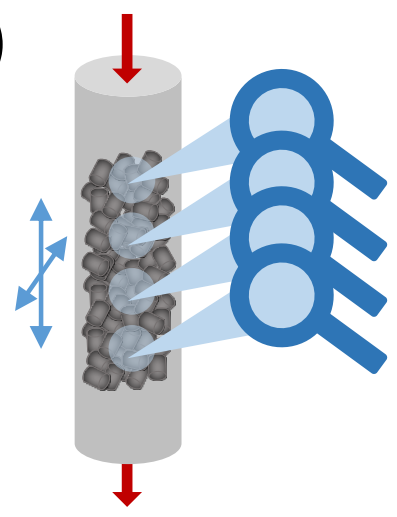

(c)

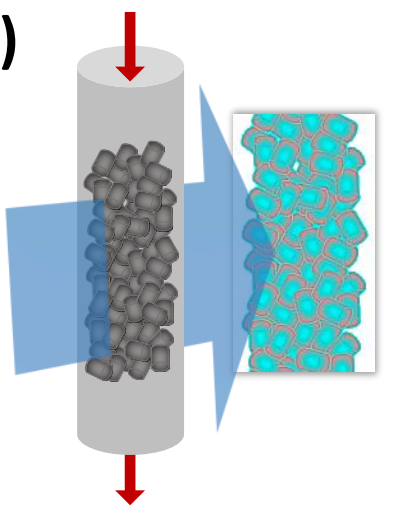

(d)

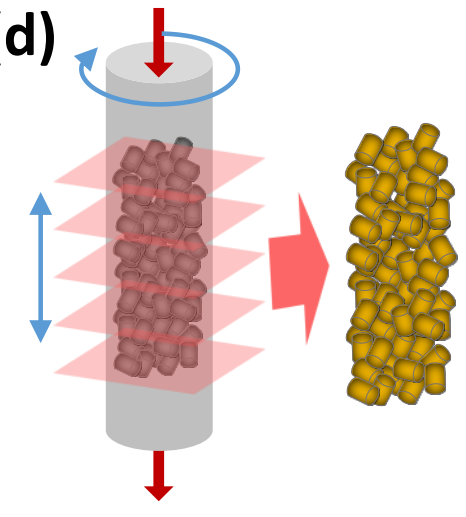

(e)
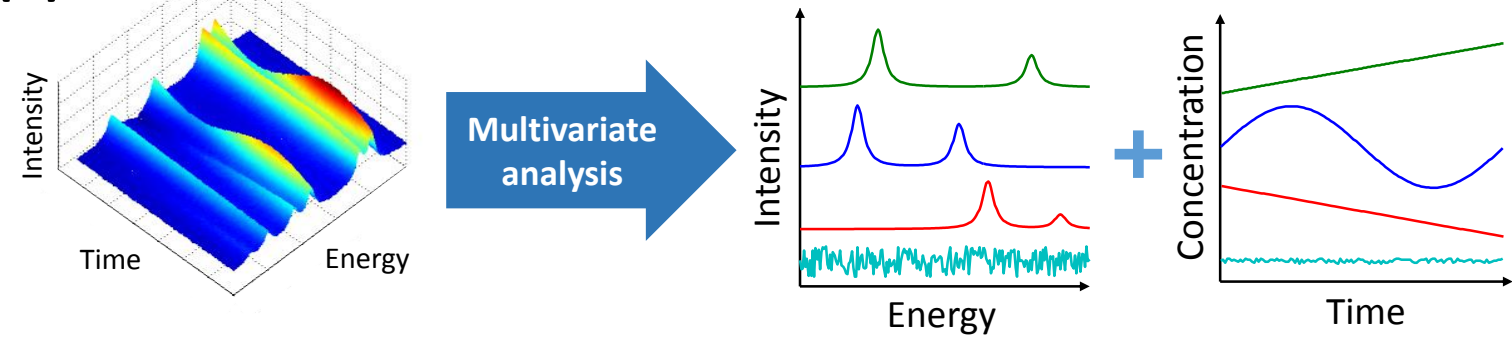